\title{
1. A Survey of Hypotheses about the Aether's Structure
}

The obvious features of the aether are: (1) its mereological structure, namely that it has parts and that some parts of the aether are parts of other parts; (2) the way parts of the aether vary in extent; and (3) the way they vary in quantity. The aim of this chapter is to survey hypotheses about these elements of structure. The survey will exhibit the great variety of the hypotheses between which I will be choosing, and so counter the blancmange prejudice, namely the assumption that the aether has no interesting structure.

The survey will also, I hope, provoke readers into provisionally judging them in an a priori way, and so overcome any empiricist scruples they might have. I say 'provoke' because it is customary not to bother with 'silly' or 'crazy' hypotheses unless you intend to defend them. But this custom covers-up our reliance upon the a priori intuitions used to reject them, as does the careless dismissal of such hypotheses on grounds of simplicity. At the point where readers exclaim, 'spare us any more hypotheses' I urge them to be honest about their reliance on the (defeasible) a priori. $^{32}$

My survey is carried out subject to certain restrictions. One of these is that extent is explicated as diameter; bearing in mind that Relativity seems to imply that diameter is frame relative. Likewise the quantity of a region will initially be explicated as its hypervolume - the 4 dimensional volume analog - although in the next chapter I shall argue that this is not always the case. These restrictions are, therefore, simplifications for the purposes of exposition.

To survey the hypotheses I use the method of coordinate representation. There are other methods, such as describing the actual structure

Recently Laurence Bonjour (1998) has defended rational insight as a source of a priori beliefs. This suggests a remarkable faculty. Possibly we have such a faculty, and if so the only explanation I find satisfactory is divine providence. But I see no need to posit it. For I hold to an epistemology in which any belief whatever is rational unless there are grounds for questioning it that due diligence either has or should have revealed. 
by noting that adjoining some fictions results in a more familiar structure. That may be compared to Hartry Field's program of science without numbers, where numbers are presented as fictional additions to the physical entities (Field 1980). Because the method of representation is easier, I shall ignore the method of fictions except for some very special cases, notably the fictional addition of the empty region.

I begin with a brief account of the mereology of the aether, partly to introduce ideas that will be needed later, but chiefly to combat the blancmange prejudice mentioned above. Although no issues will be settled in this chapter, the sheer variety of hypotheses shows that there is a question to be answered as to the mereological structure of aether and that it goes beyond the debate over whether there are aether atoms .

\section{Aether mereology ${ }^{33}$}

I begin by restricting attention to full parts of the aether. The contrast is with, for example, the putative part of the aether consisting of $50 \%$ of the aether everywhere. Ockhamist appeals to simplicity support the position that all parts of the aether are full parts, but I shall have occasion to consider the alternative in Chapter Five. In accordance with this restriction to full parts I define a region to be a full part of the aether, and concentrate on the mereology of regions. To avoid confusion, I do not call parts of Space-time regions.

Mereology concerns the part/whole relation, $\mathrm{x} \leq \mathrm{y}$. As is standard, I stipulate that everything is part of itself, $\mathrm{x} \leq \mathrm{x}$, and define proper parthood, $\mathrm{x}<\mathrm{y}$ by: $\mathrm{x}<\mathrm{y}$ if $\mathrm{x} \leq \mathrm{y}$ and $\mathrm{x} \neq \mathrm{y}$. I take it as self-evident, and no doubt analytic, that parthood is transitive, that is, a part of a part is a part, and that proper parthood is anti-reflexive.

The other axioms are metaphysical hypotheses, even if we grant their non-contingency. For instance, because of the restriction to one kind of stuff, the aether, the mereology is taken to be extensional, that is (2009). 
no two non-atomic regions can have the very same regions as proper parts. $^{34}$

An upper bound of some regions, the Fs, is defined to be any region of which every $\mathrm{F}$ is a part. A lower bound of the Fs is any region that is part of every F. Two regions without a lower bound are said to be disjoint. Otherwise they are said to overlap. One of the differences between the part/whole relation on the aether and many other orderings is that there is something, œ, the whole aether, of which every region is part, but there is no empty thing, $\varnothing$, that is part of every region. The other difference is that because the relation of being disjoint is of intuitive importance there are two rival ways of explicating the non-technical idea of the combination of two or more region into a larger one. I shall stipulate that the word 'sum' is reserved for this non-technical idea of the combination operation and not be used as synonymous with fusion, which is thus one potential explication of summation. Although the definition of a fusion of the Xs is familiar it is somewhat convoluted: a fusion of the Xs is some region that overlaps all and only the regions that overlap some $\mathrm{X}$. It is customary to speak of the fusion, assuming uniqueness. Uniqueness of fusion follows from the principle of Weak Supplementation, namely that if $\mathrm{x}$ is a proper part of $\mathrm{y}$ then there is some part $\mathrm{z}$ of $\mathrm{y}$ disjoint from x. Weak Supplementation will be discussed further below.

More straightforward than fusion is the idea of the join $\mathrm{x} \vee \mathrm{y}$ of $\mathrm{x}$ and $\mathrm{y}$, or more generally the join of the Xs, $\vee X$. It is the least upper bound, that is, an upper bound (of $x$ and y or the Xs, respectively) that is part of every other upper bound. If there is a join thus defined, then it is unique. Joins are one possible explication of sums. Likewise we may define the meet $(\mathrm{x} \wedge \mathrm{y}$, or $\wedge \mathrm{X})$ as the greatest lower bound, that is, a lower bound of which every lower bound is part. Clearly disjoint regions have no meet. It is, however, intuitive that overlapping regions always do. If there is a meet then it is unique.

Some hypotheses about mereology thing can be constituted by another, as in the lump of clay and the statue. 
One way of assuming more than the minimum amount of mereology is to make the plausible supposition that any two overlapping regions have a meet and any two regions a join. Likewise it is intuitive that both the distributivity laws hold. ${ }^{35}$ To state the laws neatly we take the meet of disjoint regions to be the fictional empty region, ø. If we adjoin this fictional region $\varnothing$, then I say we are considering a lattice of regions, reserving the term 'mereology' for the system of real regions. So a distributive lattice of regions is one in which any two members have a join and a meet and the distributive laws hold: $x \wedge(y \vee z)=(x \wedge y) \vee(x \wedge z)$ and $\mathrm{x} \vee(\mathrm{y} \wedge \mathrm{z})=(\mathrm{x} \vee \mathrm{y}) \wedge(\mathrm{x} \vee \mathrm{z})$. A mereology whose lattice is distributive will be called a distributive mereology.

Another operation of interest on a lattice is the complement $\neg \mathrm{x}$ of a region $\mathrm{x}$. This is the join of all the regions disjoint from $\mathrm{x}$. Clearly $\neg \varnothing=$ $œ$ and $\neg œ=\varnothing$. If the distributive laws hold then the complement of a join is the meet of the complements, and the complement of a meet the join of the complements. If in addition, for any $\mathrm{x}, \neg \neg \mathrm{x}=\mathrm{x}$, then a distributive lattice is said to be Boolean, as is the corresponding mereology.

We may also define the difference $\mathrm{x}-\mathrm{y}$ as the join of all the parts of $\mathrm{x}$ disjoint from $\mathrm{y}$. In a lattice $\mathrm{x}-\mathrm{y}=\mathrm{x} \wedge \neg \mathrm{y}$.

It is intuitive that the mereology of the aether is Boolean as are all the four exemplars. The cases of infinite joins and meets are not so clear.

If finite meets distribute over arbitrary joins, the mereology becomes a complete Heyting lattice if the fictional empty region $\varnothing$ is adjoined. ${ }^{36}$ I call this a complete Heyting mereology. It may be thought of as the result of weakening classical mereology by abandoning Weak Supplementation.

The necessary and sufficient condition for either of the two distributivity laws is that (even including the fictional empty region ø) there are no five regions that instantiate the mereological relations represented by the Hasse diagrams M5 and N5. As a corollary either distributivity laws implies the other (Grätzer 1971).

The categories of complete Heyting algebras, frames and locales have the same objects, which I call complete Heyting lattices, but differ in their morphisms. See (Johnstone 1982.) 
Classical mereology (restricted to regions) or general extensional mereology as it is widely called, is obtained by adjoining to the transitivity and anti-reflexivity of parthood the one, deceptively simple, axiom that any regions have a unique fusion. This implies that the unique fusion is a join. In fact it shows that if we adjoin the fictional empty region the mereology becomes a complete Boolean algebra. The principles of classical mereology imply those of complete Heyting mereology but the converse is not the case. The difference between classical mereology and (non-classical) complete Heyting mereology may be illustrated in a heuristic way by considering the case in which every region has positive diameter but there is a point particle. Then in classical mereology the particle should either be inside a given region or outside it, while in complete Heyting mereology the particle could as it were be sitting on the fence between two regions.

The case for classical mereology is based upon the intuitive principle of Weak Supplementation, namely that if $x$ is a proper part of $y$ then there is some part $\mathrm{z}$ of $\mathrm{y}$ disjoint from $\mathrm{x}$. From this it follows that the join of some regions must be a fusion, and that any fusion must be the join. Classical mereology follows from Weak Supplementation together with the principle that any regions have a fusion. Although I consider Weak Supplementation somewhat intuitive, I want to contrast it with a much firmer intuition, Interior Part Supplementation:

If $\mathrm{x}$ is an interior part of $\mathrm{y}$ then there is some part $\mathrm{z}$ of $\mathrm{y}$ disjoint from $\mathrm{x}$.

To say that $\mathrm{x}$ is an interior part of $\mathrm{y}$ is to say that $\mathrm{x}$ is separated from every region $\mathrm{z}$ disjoint from $\mathrm{y}$. Separation may in turn be characterised in the continuous case by saying that two regions $x$ and $y$ are separated if they are a positive distance apart. (See Chapter Two, Section Three, for further discussion of separation). In the next chapter I consider a premise (Premise Eight: Hypervolume Supplementation) that is more intuitive than Weak but less intuitive than Interior Part Supplementation.

Suppose we do not assume Weak Supplementation but assume the lattice obtained by adjoining $\varnothing$ is a complete Heyting lattice. Then the complement $\neg \mathrm{x}$ of $\mathrm{x}$ is defined, as above, as the join of all the $\mathrm{y}$ disjoint from $\mathrm{x}$. It follows that $\mathrm{x} \leq \neg \neg \mathrm{x}$. If $\mathrm{x}=\neg \neg \mathrm{x}$ then $\mathrm{x}$ is said to be regular. The regular members of a complete Heyting lattice form a complete Bool- 
ean lattice (although the join in the subsystem of regular regions is not always the same as the join in the system of all the regions). One way to obtain a classical mereology, then, is fictionally to identify regions $\mathrm{x}$ and $\mathrm{y}$ that are classically equivalent in the sense that $\neg \neg \mathrm{x}=\neg \neg \mathrm{y}$.

\section{Explicating summation}

Initially it is not clear whether the non-technical idea of the sum should be explicated as join or fusion, so it is worth considering the difference, if there is one. To be sure in classical mereology any regions have a unique fusion, which is their least upper bound, so there is no difference, but classical mereology, although intuitive, is defeasible.

I now argue that the sum is a join that is also a fusion. First, I note that if the join of the Xs exists then it overlaps every region that overlaps some X. Hence, if it fails to be a fusion, that is because it overlaps some region disjoint from every $\mathrm{X}$, and hence has a part disjoint from every $\mathrm{X}$, which is not what we expect from the sum of the Xs.

Next I consider an example. Because I am here considering the general concept of summation I am not required to restrict myself to the aether but I may consider Time in isolation from Space. In that case, one hypothesis we might consider is that all Time's parts are intervals, represented by sets of real numbers of the form $\{\mathrm{t}: a<t \leq b\}$, where $\mathrm{a}<\mathrm{b}$ and we allow as special cases $a=-\infty$ and $b=\infty .{ }^{37}$ Call this hypothesis Intervals Only. It generalises to the aether, where it becomes the hypothesis that all regions must be convex, but I find that much less intuitive than Intervals Only for Time. Now consider the two intervals $\mathrm{c}$ and $\mathrm{d}$ represented by $\{\mathrm{t}: 1<t \leq 2\}$ and $\{\mathrm{t}: 3<t \leq 4\}$. They have a join e represented by $\{\mathrm{t}: 1<t \leq 4\}$. But they have no fusion. Intuitively they have no sum because the sum, if it existed would have to be represented by $\{\mathrm{t}$ : $1<t \leq 2\} \cup\{\mathrm{t}: 3 t \leq 4\}$, but on Intervals Only there is no such part of Time. This example supports the requirement that the sum of regions should be a fusion.

On the other hand, it is intuitive that the sum of regions contains every one of them as parts, so the sum, if it exists, must be an upper

The use of the half-open intervals is not essential for the example. 
bound. If it is not a least upper bound then why should not some smaller one deserve to be considered a sum? Intuitively a sum, if it exists, should be unique. Joins are unique but fusions not always so. Therefore the sum should be the join. I explicate the idea of the sum, then, as a join that is also a fusion. One intuitively appealing requirement for mereology is, then, that any Fs have a sum, and hence a join that is also a fusion of the Fs. This will be defended in the next chapter. It is, however, weaker than classical mereology, which requires that fusions be unique. In fact it holds in a complete Heyting mereology, in which there can be two regions $u$ and $v$, where $u$ is a maximal proper part of $v$. In that case $u$ is a fusion of $v$ and vice versa. Rather than take this as counter-intuitive, I think we should grant that, outside classical mereology, fusion fails to explicate summation.

\section{Simples and other atoms}

Given classical mereology any region $\mathrm{x} \neq \propto$ has a unique complement. This enables us to divide $œ$ into atoms and gunk, where an atom is a part that is not the sum of two disjoint parts and any part of gunk has a proper part. In classical mereology atoms coincide with simples, that is, parts of $\propto$ with no proper parts. In that case, either there are no atoms, so aether is gunk, or the aether is the sum of atoms, in which case there is no gunk, or the aether is the sum of two portions, the sum of all atoms and the sum of all gunk. I reject the latter hypothesis on Ockhamist grounds. The only further question of mereological interest is then just how many atoms there are or how many parts to gunk. If, however, mereology is non-classical but the aether is the sum of atoms, then by definition all simples are atoms but it is an open question whether all atoms are simples.

\section{The size of a mereology}

'How big is the mereology of regions?' Here the only answers that we are tempted to, but should not, ignore are those in which there are too many parts to have a cardinal number assigned. For instance, suppose the aether, $\propto$, is a hypersphere and is made up of points (regions of zero diameter). Then each point might be an exact replica of the whole of $œ$, with its own diameter function, which might then be considered to be of 
infinitesimal amount. We might also consider the hypothesis that what we think of as the whole of aether is but a point in another, qualitatively identical region. By itself this does not provide an exotic mereology, it is gunk with no more regions than there are real numbers. Now, however, let us go further and suppose that for every limit ordinal $\alpha$ there is a point corresponding to every sequence of 'worlds within worlds' indexed by the ordinals less than $\alpha$. The resulting hypothesis is one of hypergunk, (Nolan 2004, Hazen 2004) in which there is an absolute infinity of regions, that is, more than that of any set.

\section{A survey of hypotheses about mereology}

Even before we consider diameters and volumes we have, then, quite a variety of hypotheses about the structure of the aether. Of these the ones that I take to be most significant are hypotheses about gunk versus atoms, and about the number of regions along with the following hypotheses, which I have already described.

Distributive mereology: If we adjoin $\varnothing$, any two regions have a join and a meet and the distributivity principles hold.

Boolean mereology: A distributive mereology, whose lattice is Boolean

Summation mereology: A lattice mereology in which any regions have a join that is also a fusion.

Complete Heyting mereology: A summation mereology where the following infinite distributivity principle holds in the lattice:

For any $\mathrm{x}$ and any Fs, the meet of $\mathrm{x}$ with the join of the Fs is the join of the meets of $\mathrm{x}$ with individual Fs

Classical mereology: A mereology for which any regions have a unique fusion, so if $\varnothing$ is adjoined the lattice is complete Boolean. $^{38}$

The initially plausible hypothesis that classical mereology holds for the aether does not imply that it holds more generally. I have suggested that sets can be replaced by pseudo-sets, which have as their pseudo-members parts. Thus, $\{b\}^{*}$, the pseudo-singleton of $b$, has $b$ as a proper part that has every proper part of $\{b\}^{*}$ as a part, and so there is no part $x \neq\{b\}^{*}$ such that $\{b\}^{*}$ is the sum of $b$ and $x$ (Forrest 2002). 
A classical mereology must be complete Heyting and Boolean. A complete Heyting mereology must be a summation mereology. And a summation mereology must be a distributive mereology. A Boolean mereology that is also a summation mereology must be classical. A summation mereology that satisfies Weak Supplementation must be classical.

All these hypotheses about mereology have curmudgeon variants, obtained from the same lattices but with the qualification that not only $\varnothing$ but also some joins of disjoint regions are fictional. These fictions include all the joins of the regions that do not touch. The intuition behind these curmudgeon variants is that if a 'region' is disconnected then 'it' is in fact many regions, not one. In the next chapter I undermine this intuition. I also consider that this intuition is defeated by the following Scattered Object Argument. The first premise is that there are scattered material objects. The second is that a scattered object is constituted by a scattered region. So there are scattered regions. Although quantum theory clouds our understanding of such things, I claim that familiar objects, including ourselves, are scattered, consisting of nuclei that never touch each other, together with electrons that never touch the nuclei. This supports the first premise. The second premise could be rejected on the grounds that a material object is constituted by many regions not a single one. To that I reply that this introduces a mysterious mode of constitution to be contrasted with the way in which a single region constitutes a material object. ${ }^{39}$

The Scattered Object Argument is not totally persuasive, so it is worth bearing in mind curmudgeon variants. Of the exemplars, one, Arntzenius Continuum has no curmudgeon variant, because it implies that there are regions without connected parts. (See Chapter Two.) So curmudgeons should reject rather than modify it. Otherwise, all the regions are sums of connected ones and curmudgeons may treat them as fictions. Apparent reference to disconnected regions may then be paraphrased as plural reference to the connected parts.

Another family of hypotheses is obtained by proposing that a countable family of regions has a join and a meet but not all uncountable accidental properties of the region are essential properties of the object 
families do. The case for the existence of arbitrary sums is based on the intuition that unrestricted principles are more plausible than restricted variants. I note that as a reason for preferring unrestricted summation.

I list these hypotheses because they are supported by distinct intuitions, but the reader might supplement the list using Peter Simon's thorough discussion (1987). My purpose is to emphasise the variety of hypotheses, and so open up the question of the mereological structure of aether, rather than taking it for granted that classical mereology holds. As far as I can see, the case for classical mereology is based on two premises: (1) Weak Supplementation, which is intuitive but not as firmly so as the analog based on interior parthood, mentioned above; and (2) Universal Summation, which has an advantage over Countable Summation, but only because unrestricted rules are simpler than restricted ones. The resulting support is genuine but not especially strong.

\section{Coordinate representation}

If the aether has the structure of a manifold, it is the join of suitable overlapping regions each of which has a coordinate representation, but there may not be a representation of the whole aether. ${ }^{40}$ If, however, we are concerned only with the local structure of the aether that complication is irrelevant. So, one way of examining this local structure is to represent regions by sets of quadruples. I require the diameter and hypervolume of a region to be approximately that of the representing set. This mention of diameters and hypervolumes might alarm readers for neither is fundamental to the description of regions. Diameter is frame-relative, and hypervolume may be replaced by the greater-hypervolume-than ordering. But my initial aim is to use our intuitions about hypervolume and diameter in order to rank hypotheses, delaying their further discussion

I shall have more to say about manifolds in Chapters Four and Five. A 4 dimensional aether-manifold is the sum of (mereo-) topologically open regions $u_{j}, j=1,2$ etc, where, for each $j, u_{j}$ is represented by an open subset of $\mathfrak{i} 4$, the topological space of all quadruples of reals, in such a way that the open parts of $u_{j}$ are in one to one correspondence with some of the open subsets of $\mathfrak{i} 4$. We may always suppose that for each $\mathrm{j}$, $\mathrm{u}_{\mathrm{j}}$ is represented by the whole of $\mathfrak{1} 4$. 
until Chapter Four. That is quite compatible with diameter and hypervolume being derived from more fundamental relations. To be sure, the diameter depends on which relativistic frame is used, but that is of no concern in this chapter because we are representing regions using coordinate quadruples - any such representation already requires a choice of coordinate axes, and hence a frame.

Concentrating on the local structure if necessary, I take the aether, $œ$, to be represented using some or all the coordinate quadruples in some open convex set $\mathrm{E}$, so $\mathrm{E} \subseteq \Re 4$ the set of all quadruples of real numbers. Readers may choose to consider the special case in which $\mathbb{E}=\mathfrak{R} 4$, with no loss of generality relevant to this chapter.

$\Re 4$ is equipped with a norm, that is, the distance from $\langle 0,0,0,0\rangle$, defined by $\|<\mathrm{t}, \mathrm{x}, \mathrm{y}, \mathrm{z}>\|=\sqrt{ }\left(\mathrm{t} 2+\mathrm{x}^{2}+\mathrm{y}^{2}+\mathrm{z}^{2}\right)$, and with the Lebesgue measure $\mu$. I use the technical phrase 'Lebesgue measure' in place of 'hypervolume' because I am considering sets of quadruples rather than regions. The basic idea is that the Lebesgue measure of a set of quadruples is the hypervolume of a region in a fictitious 4 dimensional Euclidean space whose points are represented using Cartesian coordinates $t, x$, $\mathrm{y}$ and $\mathrm{z} .{ }^{41}$

The distance between quadruples $\xi$ and $\eta$ is $\| \xi$ - $\eta \|$. If $X \subseteq \Re 4$, then $|X|$, the diameter of $X=\sup \{\|\xi-\eta\|: \xi \in X, \eta \in X\}$, that is, is the least upper bound of $\{\|\xi-\eta\|: \xi \in X, \eta \in X\}$ or $+\infty$ if there is no upper

41 More formally, the Lebesgue measure $\mu$ is uniquely characterised as follows:

1. If $\mathrm{a}, \mathrm{b}, \mathrm{c}, \mathrm{d}, \mathrm{e}, \mathrm{f}, \mathrm{g}$ and $\mathrm{h}$ are real numbers with $\mathrm{a} \leq \mathrm{b}, \mathrm{c} \leq \mathrm{d}, \mathrm{e} \leq \mathrm{f}, \mathrm{g} \leq \mathrm{h}$ then $\mu(\{<\mathrm{t}, \mathrm{x}, \mathrm{y}, \mathrm{z}>\mathrm{a} \leq \mathrm{t} \leq \mathrm{b}, \mathrm{c} \leq \mathrm{x} \leq \mathrm{d}, \mathrm{e} \leq \mathrm{y} \leq \mathrm{f}, \mathrm{g} \leq \mathrm{z} \leq \mathrm{h}\})=(\mathrm{b}-\mathrm{a})(\mathrm{d}-$ $\mathrm{c})(\mathrm{f}-\mathrm{e})(\mathrm{h}-\mathrm{g})$.

2. The Lebesgue measure is a non-negative real number or $+\infty$

3. If $X \subset Y$ and $X$ and $Y$ have Lebesgue measures then $\mu(X) \leq \mu(Y)$.

4. If $X \subset Y \subset Z$ and if $X$ and $Z$ have the same Lebesgue measure then $Y$ has a Lebesgue measure.

5. Lebesgue measure is countably additive on pairwise disjoint sets. That is, for any countable sequence $X_{m}$ of sets of quadruples such that $X_{m} \cap X_{n}=$ $\varnothing$ if $\mathrm{n} \neq \mathrm{m}, \mu\left(\cup \mathrm{X}_{\mathrm{m}}\right)=\Sigma \mu\left(\mathrm{X}_{\mathrm{m}}\right)$, provided all the $\mathrm{X}_{\mathrm{m}}$ have a Lebesgue measure. 
bound. It follows that if $\xi$ is a quadruple, $\|\xi\|=|\{\xi,<0,0,0,0>\}|$, so unless $\xi=<0,0,0,0>\|\xi\| \neq|\{\xi\}|$.

$\Re 4$ has a topology, namely a family of open subsets $\mathrm{T}$ of $\Re 4$ containing $\varnothing$ and $\Re 4$ itself, such that $\mathrm{T}$ is closed under the operations of finite intersection and arbitrary union. $\mathrm{T}$ is specified as follows: a set $\mathrm{U}$ is open just in case, for any $\xi \in U$ there is some positive number $\varepsilon$ such that if $\|\eta-\xi\|<\varepsilon$ then $\eta \in \mathrm{U}$. A closed set is one whose complement is open. It follows that both the empty set $\varnothing$ and the set of all quadruples, $\mathfrak{R} 4$ are both open and closed. No other sets of quadruples have that distinction. The closure of a set is the intersection of all the closed sets that include it, and the interior of a set is the union of all the open sets included in it. The closure of any set is closed and the interior of any set is open. A set is said to be regular open (also called perfectly open) if it is the interior of its closure. A non-empty open set is said to be connected if it is not the union of two disjoint non-empty open sets. So $\Re 4$ is itself connected.

A representation of the aether is a mapping $\Phi$ assigning to any region $\mathrm{u}$ one or more non-empty sets of quadruples of real numbers $\Phi(\mathrm{u})$. For the moment I consider the case in which the representation is a single-valued function, that is, it assigns a single set of quadruples to a region. I require that $\operatorname{diam}(\mathrm{u}) \approx|\Phi(\mathrm{u})|$. I require that if $\mathrm{u}$ has a volume and if $\Phi(u)$ has Lebesgue measure $\mu(\Phi(u))$, then hvol $(u) \approx \mu(\Phi(u))$. Here di$\operatorname{am}(\mathrm{u})$ is the diameter of $\mathrm{u}$ and hvol(u) is the hyper-volume of $\mathrm{u}$, the 4 dimensional analog of ordinary volume. The symbol ' $\approx$ ', used for approximate equality, is required in place of " $=$ " for two reasons. The first and most straightforward is that if the aether is curved there will be a distortion due to the curvature. We may restrict attention to a region chosen so that this is no more than $1 \%$ and largely ignore this distortion as irrelevant. The second is more serious. Something peculiar might go on when we reach the Planck barrier. Suppose $K \approx 1044$, so $(1 / K)$ sec is of the order of magnitude of Planck time. For instance Point Discretion might be correct with some $\mathrm{K}^{4}$ points in a hypercube of side one (light) second. In that case there could be further distortion so that, in (light) second units, $\operatorname{diam}(\mathrm{u})$ is within $1 \%$ of $|\Phi(\mathrm{u})| \pm 1 / \mathrm{K}$. Likewise we might 
suppose that hvol(u) is within $1 \%$ of $\mu(\Phi(\mathrm{u})) \pm 1 / \mathrm{K}^{4}$. The details do not matter much and I continue to write $\operatorname{diam}(\mathrm{u}) \approx|\Phi(\mathrm{u})|$ and hvol$(\mathrm{u}) \approx$ $\mu(\Phi(u))$.

The representation should preserve the part/whole structure: for all parts $\mathrm{u}, \mathrm{v}$ of $œ$, if $\mathrm{u} \leq \mathrm{v}$ then $\Phi(\mathrm{u}) \subseteq \Phi(\mathrm{v})$ We should not assume, however, that the representation is faithful, that is a one to one correspondence, in the sense that if $u \neq v$ then $\Phi(u) \neq \Phi(v)$. For if there are points $u$ and $\mathrm{v}$ an infinitesimal distance apart then there is a coordinate quadruple $<\mathrm{t}, \mathrm{x}, \mathrm{y}, \mathrm{z}>$ such that $\Phi(\mathrm{u})=\Phi(\mathrm{v})=\{<\mathrm{t}, \mathrm{x}, \mathrm{y}, \mathrm{z}>\}$.

Even if it is faithful, the coordinate representation might not preserve joins and meets. The most familiar example is Tarski Continuum, the hypothesis that the regions are represented faithfully by precisely those sets that are non-empty regular open sets of coordinate quadruples (Tarski 1956). One of the nice features about Tarski Continuum is that the regions satisfy the principles for classical mereology because any regions, even infinitely many, have a join and that join is the unique fusion of the regions. In fact for any non-empty set of regions $\mathrm{W}, \Phi(\vee \mathrm{W})$ is the interior of the closure of $\cup\{\Phi(\mathrm{w}): \mathrm{w} \in \mathrm{W}\}$. But the representation does not always preserve joins, as the following example shows. In this case we suppose the aether $œ$ is infinite and the set representing it, $\mathbb{E}$, is the set of all quadruples, but the example copuld be restricted to the case in which $\mathrm{E}$ is an open ball of coordinate quadruples. The set of quadruples has a left open half $\mathrm{L}=\{<\mathrm{t}, \mathrm{x}, \mathrm{y}, \mathrm{z}>$ : $\mathrm{x}<0\}$ and a right open half $\mathrm{R}$ $=\{<\mathrm{t}, \mathrm{x}, \mathrm{y}, \mathrm{z}>\mathrm{x}>0\}$ with a hyperplane $\mathrm{H}=\{<\mathrm{t}, \mathrm{x}, \mathrm{y}, \mathrm{z}>\mathrm{x}=0\}$ separating them. So $\mathbb{E}=\mathrm{L} \cup H \cup \mathrm{R}$ Given Tarski Continuum, L represents a region $\mathrm{u}, \mathrm{R}$ represents a region $\mathrm{v}$ and $\mathrm{H}$ fails to represent, because it is not open. The interior of the closure of $L \cup R$ is $L \cup H \cup R=\mathbb{E}$. So $\propto=u \vee v$, even though $\mathbb{E}=\mathrm{L} \cup \mathrm{H} \cup \mathrm{R} \neq \mathrm{L} \cup \mathrm{R}$. A similar result holds if all regions are represented by sets of quadruples of 4 dimensions. Both these hypotheses violate Weak Supplementation, as Hud Hudson notes (2005: 50-56).

Should we prefer hypotheses with greater restrictions on regions? In that case we should prefer Tarski Continuum to the hypothesis that the regions correspond to all non-empty open sets, which in turn should be preferred to the hypothesis that they correspond to all four-dimensional regions. And in that case all these hypotheses should be preferred to the 
Orthodoxy that all non-empty sets correspond to regions. Or should we argue that the fewer and simpler the constraints the better? That would support the Orthodoxy. Neither, I say! For both ways of arguing assume there exist possible points corresponding to all coordinate quadruples and that our hypothesis is about which of these possible points are actual. We should not forget that the sets of coordinate quadruples are representations of the parts of $\propto$ and so hypotheses about these sets do not describe the intrinsic structure of the aether. The criteria of simplicity apply to the intrinsic structure not to the way that structure is represented.

\section{The Axiom of Choice and Banach Tarski.}

The Axiom of Choice is intuitive, as shown by the way that it requires practice even to notice its use. But the occurrence of non-measurable sets (e.g. sets of coordinate quadruples lacking any Lebesgue measure, even 0 or $\infty$ ) is also somewhat surprising, and Solovay has shown that if set theory with the Axiom of Choice is consistent then so is set theory in which there are no non-measurable sets (1970). I ask, then, how rejecting the Axiom of Choice would affect the investigation of the structure of the aether. Consider, for instance, the Orthodoxy that any non-empty set of coordinate quadruples represents one and only one region, and so, in particular, every singleton represents an point. Given realism about sets of quadruples, there is a fact of the matter as to whether the Axiom of Choice holds. If it does, then the celebrated Banach Tarski theorem also holds. ${ }^{42}$ Hence there are disjoint regions b1, b2, b3, b4, b5; c1, c2, $\mathrm{c} 3, \mathrm{c} 4$, and $\mathrm{c} 5$ such that each $\mathrm{bj}_{\mathrm{j}}$ is represented as having the same shape and diameter as the corresponding $\mathrm{c}_{\mathrm{j}}$, and yet the join of the $\mathrm{bj}_{\mathrm{j}}$ is a ball of $1 \mathrm{~cm}$ radius lasting for 1 second while the join of the $\mathrm{cj}$ is a ball of 2 $\mathrm{cm}$ radius lasting also for 1 second. This result implies that not all regions have a hypervolume, provided we assume: (1) that regions that are represented as congruent have approximately equal hypervolume - within $5 \%$ is far more accurate than required; and (2) that the hypervolume 
of the join of finitely many disjoint regions is the sum of their hypervolumes. $^{43}$ For in that case, if all the regions have a hypervolume then that of the ball lasting one second would equal that of one of twice the radius, to within $5 \%$, which is absurd. The assumption that regions represented by congruent sets of quadruples have volumes within $5 \%$ follows from the requirement that the diameters are represented to within $1 \% \pm$ $1 / \mathrm{K}$, and the obvious assumption that $\mathrm{K}$ is greater than 1000 . For then the error in hypervolumes is less than $(1.1) 4 \%$, which is less than $5 \%$. Curmudgeons who insist all regions are connected may still obtain regions to which no hypervolume can be assigned. They will differ from $\mathrm{b}_{1}, \mathrm{~b}_{2}, \mathrm{~b}_{3}, \mathrm{~b} 4, \mathrm{~b} 5$; c1, c2, c3, c4, and $\mathrm{c}_{5}$ by regions of zero hypervolume, but they will not be disjoint.

The Banach Tarski theorem does not hold if we suppose Solovay's Axiom in place of the Axiom of Choice. In that case the Orthodoxy implies that we may assign a hypervolume to any region, as we intuitively expect. The Orthodoxy then has an additional intuitive advantage over the two continuous exemplars, Borel and Arntzenius Continuum. For on the Orthodoxy all regions are composed of aether points using the one basic operation of summation. By contrast, on the hypotheses of Borel and Arntzenius Continuum regions are composed of fundamental regions ('globules') using two basic operations, summation and difference.

Initially, then, the Orthodoxy combined with Solovay's Axiom might well be taken as a serious contender for the structure of the aether, and defeat the Axiom of Choice. I have, however, two reasons for rejecting this defeater, leaving the Axiom of Choice as a defeasible but undefeated intuition.

My first reason for taking the Axiom of Choice to be undefeated is that the case for there being some non-measurable sets, and hence the case against Solovay's Axiom requires only a special, and especially in-

Typically we suppose that (hyper)volume is represented by Lebesgue measure, which satisfies countable additivity. It is noteworthy, therefore, that the Banach Tarski theorem only requires (hyper)volume to be finitely additive, which is intuitively much more secure than countable additivity. 
tuitive, instance of the Axiom of Choice. ${ }^{44}$ For that axiom has been assumed at just one point in the argument, namely that given a suitable equivalence relation there exists a cross section - a set that contains just one member from each equivalence class. To assume that for any equivalence relation we could find a cross-section would be tantamount to assuming the Axiom of Choice itself. But the especially intuitive case being considered is that in which the equivalence relation is 'natural' in sense that Lewis uses that term, as opposed to 'artificial'. (Here an artificial term is one that would not be used to state a hypothesis if we are to assess its simplicity.)

The other reason why the Axiom of Choice is an undefeated intuition is that its potential defeater, the Orthodoxy without the Axiom of Choice, is not as attractive as might initially appear. The Orthodoxy attracts us because, if it is correct, all regions are sums of points, which permits the assertion that all other regions depend for their existence on points. Because points may reasonably be taken to be simples this is in accordance with the thesis that everything depends ontologically on simple things, their properties, and their relations. That may in turn be

We could use the proof of the Banach Tarski theorem to illustrate this but an easier result due to Giuseppe Vitali suffices to show where an instance of the Axiom of Choice is assumed. Consider a circle, of unit radius, and say that two points are equivalent if one may be obtained from the other by a rotation by a whole number of radians. That is, two points on the circle are equivalent if for some integer $\mathrm{n}$, there is a path of length $\mathrm{n}$ going around the circle, perhaps many times, connecting the two points. Now consider a set $\mathrm{X}$ containing just one member of each equivalence class. For any integer $n$, let $X(n)$ be the result of rotating $X$ by $n$ radians. Because there are $2 \pi$ radians in a full circle and because $\pi$ is irrational, $X=X(n)$ if and only if $n=0$. The whole circle is the union of all the $\mathrm{X}(\mathrm{n})$. If $\mathrm{X}$ has a countably additive measure invariant under rotation, such as Lebesgue measure, then the whole circle has length that is the sum of countably many equal quantities, namely the measures of the $X(n)$. Let this measure be $\eta$. Then $2 \pi$ is a countable infinity times $\eta$. This is impossible. For a countable infinity times $\eta$ is the least upper bound of all $\mathrm{N} \eta$ for positive whole numbers $\mathrm{N}$. If $\eta=0$ (or infinitesimal) the least upper bound is 0 (or infinitesimal, respectively). Otherwise it is infinity, but in neither case can it be $2 \pi$. 
argued for by means of the intuitive premises that (1) every composite thing depends on the parts of which it is composed and how they are related, and (2) there cannot be an infinite regress of dependence relations. (This is the less obscure half of Kant's Second Antinomy.) Now suppose we reject the Axiom of Choice in order to save the Orthodoxy. Then we should rely on the intuition that there is a measure of the quantity of every region, namely hypervolume. For it was only the occurrence of nonmeasurable sets that might have made us reject that intuition. Within the scope of that intuition we have a further intuition expressed $b$ the following principle.

\section{Dependent Quantity:}

If something depends ontologically on some disjoint parts then its quantity is independent of the relations between those parts. Suppose, then, that $\mathrm{b}$ and $\mathrm{c}$ depend ontologically on some disjoint parts, the Bs and the Cs, respectively, all of which are intrinsic duplicates. And suppose there is a one to one correspondence between the Bs and Cs. Then the quantity of $b$ should equal that of $c$. So if regions depend ontologically on the point parts of a continuous aether, we may derive a conclusion known to have been false at least as far back as Duns Scotus, namely that regions whose points are in one to one correspondence have the same quantity (Solère 2010: 5-6.) Scotus used the example of two concentric circles. To be sure the intuitions relied upon in this argument are not very firm but they are strong enough to undermine the case against the Axiom of Choice.

In addition, I note that thesis that all regions are of maximal dimension, which I am taking to be 4, itself has some initial appeal in the context of continuous aether. In the next chapter I undermine that intuition, replacing it with the thesis that all regions depend on those of 4 dimensions. While I do not attach much weight to that intuition I note that when combined with the thesis that everything exists in virtue of simple things, their properties, and their relations, it completes the antinomy and, for what it is worth, weighs against continuous theories of the aether rather than supporting the Orthodoxy. 


\section{Some examples of the method of representation}

The method of representation enables us to state various hypotheses about which sets of quadruples do the representing. Here are some examples, starting with the exemplars, the first two of which are discrete hypotheses. $^{45}$

\section{Point Discretion}

The most straightforward case, Point Discretion, is that in which the aether is the sum of points that are represented by the quadruples of integers. We can generalise, although that case suffices for the discrete ana$\log$ of Special Relativity. More generally, then, we suppose there is a set of quadruples $\mathrm{D}$ and a positive number $\delta$ such that if $\xi \in \mathrm{D}, \eta \in \mathrm{D}$ and $\xi$ $\neq \eta$ then $\|\xi-\eta\|>\delta$. It follows that if $\mathrm{D}$ is infinite then it is countable. The hypothesis is that all and only the non-empty subsets of $\mathrm{D}$ represent regions.

A variant on Point Discretion would be to consider, for instance, points represented by the quadruples of rational numbers in $\mathrm{E}$. More generally, the points may be represented by a set of quadruples $D^{*}$ that is countable but for which there is no positive number $\delta$ such that if $\xi \in$ $D^{*}, \eta \in D^{*}$ and $\xi \neq \eta$ then $\|\xi-\eta\|>\delta$. In fact we might assume that $D^{*}$ is dense in $\mathrm{E}$, that is, its closure is the whole of $\mathbb{E}$ as in the case of the quadruples of rational numbers.

Another variant is the infinite dimensional one in which points are represented by sequences of real numbers, for instance by infinite sequences all but finitely many members of which are zero.

\section{Granulated Aether}

By Granulated Aether I mean Extended Simples and its variants. On these hypotheses there are said to be significant small (presumably Planck scale) regions, the granules, represented using a set $\mathrm{C}$ of pairwise dis- 
joint, non-empty open connected subsets of $\mathrm{E}^{46}{ }^{46}$ ft follows that $\mathrm{C}$ is either finite or countably infinite. Extended Simples states that all and only the unions of members of $\mathrm{C}$ represent regions. So every region is the sum of extended simples, which are the granules. ${ }^{47}$ In that case the aether is connected if the interior of the closure of the union of $\mathrm{C}$ is connected.

Is the aether that constitutes our universe connected? I have no objection to island universes not connected to our part of the universe. It is then a matter for stipulation whether we consider them parts of our universe. I stipulate that they do not. The variety of disconnectedness that concerns my investigation into the structure of the aether is that which might occur at some small, presumably Planck, scale. We could, for instance, hypothesise aether simples that are represented by sets of diameter $1 / 2$ whose centres are quadruples of integers. So there would be gaps between them. Either this would just be a silly way of representing Extended Simples or it would imply the triviality of the topological relation of adjacency (touching), because no two simples would touch. Assuming we have need of topology that would be a disadvantage. So in neither case need we bother with this variant.

Some readers might prefer to represent aether simples as the sets of quadruples that are the closures of the members of $\mathrm{C}$, but because all we

A non-empty open set is said to be connected if it is not the union of two disjoint non-empty open sets.

As Varzi (2009) notes, several philosophers have recently defended the idea of simples that are extended not just in time but in Space. In particular I note Ned Markosian (1998), who, however, argues for the principle that every maximally connected thing is a simple, which would have the unhappy consequence that the aether has no proper parts. Simons (2004) defends simples from the Geometric Correspondence Principle (Simons 2004: 372), which I shall discuss in Chapter Three. David Braddon-Mitchell and Kristie Miller (2006) propose a connection between extended simples of what I call the aether and contemporary physics. See also Hudson (2006), Kris McDaniel (2007a), and Ted Sider (2007). One of the aims of this work is to present a disjunction between Extended Simples and a symmetric continuous aether hypothesis. I shall argue, however, that contemporary physics tends to support the latter. 
are doing is representing regions there is no genuine difference between theories corresponding to the choice of representations. For the difference, if there were one, would concerns the boundaries of the aether simples, but a boundary would be a proper part, and so there are no boundaries of simples.

Of special interest is the case in which the members of $\mathrm{C}$ are polytopes, that is, higher dimensional analogs of polyhedra. In that case I say the aether simples are polytopic. For instance they might be represented by the 4 dimensional analogs of tetrahedra, the pentatopes. Diagram One illustrates the difference between Point Discretion and Extended Simples in a 2 dimensional case in which the extended simples are represented as right-angled triangular regions. ${ }^{48}$

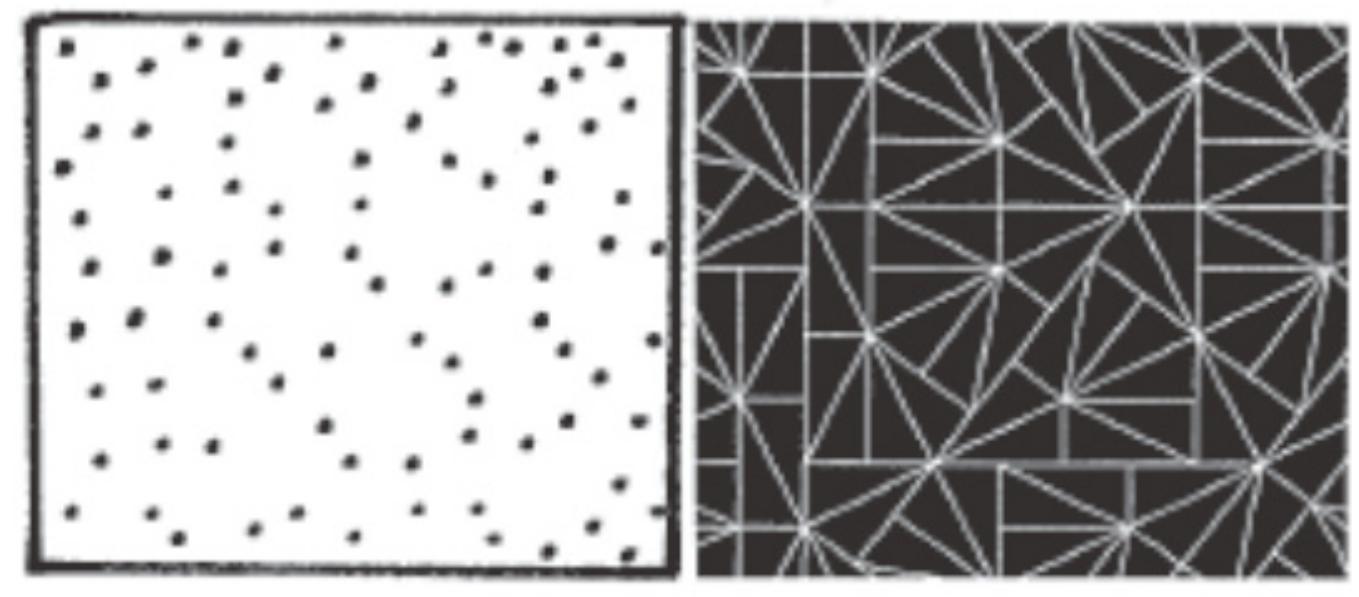

\section{Diagram One}

The most interesting variant on Extended Simples is Pseudo-set Granules, a hypothesis in which closed cells represent atoms but these atoms are not simple because the hyperfaces, faces edges and vertices of the cells also represent atoms, with only the vertices being simple. ${ }^{49}$ This

48 Based on the Pinwheel Tiling (Radin 1995),

I call this Pseudo-set Granules, because we could develop a theory of sets, or as I called them pseudo-sets, in which the pseudo-singleton of $u$ has $u$ as its greatest proper part (Forrest 2002). On this theory the granule is a pseudosingleton whose 'member' is the sum of all its hyperfaces. 
fails to satisfy Weak Supplementation, but will turn out to have compensating attractions.

\section{Borel Continuum}

The Borel subsets of $\mathrm{E}$ are the members of $\mathrm{B}$, where $\mathrm{B}$ is the intersection of all those subsets $\mathrm{X}$ of $\mathrm{E}$ that contain the open sets and where $\mathrm{X}$ is closed under the operations of countable union and the taking of complements. It follows that B contains all closed sets. Every singleton is closed, so B contains every finite and every countably infinite set of coordinate quadruples. One of the important features of Borel sets is that a Lebesgue measure (representing hypervolume) can be assigned to them. So the weird division used in the Banach Tarski theorem involves sets that are not Borel. The Borel Continuum is the hypothesis that all and only the non-empty Borel sets represent regions.

The Borel Continuum is a hypothesis in which there are points, that is, point regions. Variants that likewise have points are the Orthodoxy that all the non-empty set of quadruples represent regions, and the Lebesgue Continuum that all and only the non-empty measurable sets represent regions. Assuming the Axiom of Choice, I have noted that the Orthodoxy suffers from Banach Tarski trouble, and that neither the Borel nor the Lebesgue Continuum obey the principle of arbitrary fusion. They do, however, satisfy Weak Supplementation and countable fusion.

Another variant on Borel Continuum is that in which the regions are represented by all non-empty $\mathrm{G} \delta$ sets that is, countable intersections of open sets. All G $\delta$ sets are Borel sets and they are of mathematical interest because every Lebesgue measurable set differs from a $\mathrm{G} \delta$ set by a set of measure zero. I reject this hypothesis of $\mathrm{G} \delta$ Continuum as inferior to Borel Continuum for two reasons, both to do with taking complements. The first is that G $\delta$ Continuum is not Boolean. For we will be able to find a region $u$ such that for any region $v$ disjoint from $u$ there will be a region $w$ disjoint from $u$ of which $v$ is a proper part. ${ }^{50}$ The se-

To prove this first note that the intersection of two $\mathrm{G} \delta$ sets is a G $\delta$ set. Therefore if $\mathrm{v}$ is disjoint from $\mathrm{u}$ the representing sets of coordinate quadruples $\mathrm{V}$ 
cond is that if G $\delta$ Continuum held then complex regions would be composed of simpler ones as meets. Initially meets might be thought no worse than complements as a way in which some regions depend on others. But, although neither taking complements nor countable meets is an initially intuitive way of grounding some regions in others, a case can be made for taking complements, provided we are realists about absences. No metaphysician should take absences as fundamental entities, but the idea of the absence of $u$ depending on region $u$ is fairly intuitive. Then the difference $u-v$ can be understood as the result of combining $u$ with the absence of $\mathrm{v}$, and, in particular $\neg \mathrm{v}=\propto-\mathrm{v}$ is a combination of the whole of the aether with the absence of $\mathrm{v}$. The intuitive idea here is that the absence of $v$ 'eats up' a v-shaped hole in the aether, so adding the absence of $v$ subtracts $v$.

\section{Arntzenius Continuum}

This hypothesis differs from the previous ones in that it is most perspicuously stated as a many-valued representation. First we consider an equivalence relation on the Borel sets $\mathrm{B}$, that of almost identity. This use of 'almost' is standard in mathematics and means that something holds except on a set of measure zero. So $\mathrm{X}$ is almost identical to $\mathrm{Y}$ if they differ by a set of Lebesgue measure zero, that is if $\mathrm{X}-\mathrm{Y}$ and $\mathrm{Y}-\mathrm{X}$ are both of zero measure. Arntzenius Continuum is obtained by representing the regions by equivalence classes of Borel sets. That is, Borel sets $\mathrm{X}$ and $\mathrm{Y}$ represent the same region just in case they differ by a set of measure zero. All and only the sets of positive measure in B represent regions. The restriction to sets of positive measure is required because a set of zero measure is equivalent to $\varnothing$ and so would represent the fictional empty region $ø$.

and $\mathrm{U}$ respectively will have empty intersection. If $\mathrm{V} \subset \mathrm{E}-\mathrm{U}$, there is some point $\mathrm{p}$, represented by quadruple $\xi$, in neither $\mathrm{u}$ nor $\mathrm{v}$. Then $\mathrm{W}=\mathrm{V} \cup\{\xi\}$ is a $\mathrm{G} \delta$ set representing $\mathrm{w}$ and $\mathrm{w}$ is disjoint from $\mathrm{u}$. It follows that the required result holds provided there is a (non-empty) $G \delta$ set $U$ such that $E-U$ is not itself a $\mathrm{G} \delta$ set. For example, let $U$ be the set of all quadruples whose first coordinate is irrational. 
Every measurable set differs from a Borel set by measure zero and so we could as easily have represented the regions as equivalence classes of measurable sets. ${ }^{51}$ Using the Axiom of Choice, we could also have selected just one Borel set from each equivalence class, with $\varnothing$ selected from the class of sets of zero measure, to obtain a set $\mathrm{B}^{*}$ that represents the regions using a single-valued function, but that is messy.

To characterise the representation we also need to define the diameter and hypervolume of an equivalence class $\mathrm{Z}$ of Borel sets, which must approximate that of the region represented. Since every member of $Z$ has the same measure, the hypervolume of $Z$ is just the measure of any member of $Z$. The diameter of $Z$ is the greatest lower bound of the diameters of members of $Z$. That is, we may ignore any set of measure zero when considering diameters.

The representation of Arntzenius Continuum is more complicated than that of the other hypotheses, but it would be a mistake to infer that it is a complicated hypothesis. It has the slight advantage over Borel Continuum of being a classical mereology and has the advantage over the Orthodoxy of assigning hypervolumes to all regions. It has the further advantage over Borel Continuum that hypervolume is a faithful measure of quantity. That is, there are no two regions whose difference is of zero hypervolume.

This last advantage needs to be understood correctly, because I have deemed that the infinitesimal is zero. Consider, then, the situation in a Borel Continuum in which there are regions $u$ and $v$ with $u$ a proper part of $\mathrm{v}$ such that $\mathrm{v}-\mathrm{u}$ has 'zero' hypervolume. ${ }^{52}$ That could be interpreted as a case in which $v-u$ is of infinitesimal hypervolume. The advantage of Arntzenius over Borel Continuum may then be expressed as a dilemma facing Borel Continuum. Either the measure of quantity fails to record any difference between $u$ and v, because 'zero' means 'literally zero'

51 In fact the measurable sets may be characterised thus: If $\mathrm{W}$ is measurable there are Borel sets $\mathrm{X}, \mathrm{Y}$ and $\mathrm{Z}$ such that $\mathrm{W}-\mathrm{X} \subseteq \mathrm{Y}, \mathrm{X}-\mathrm{W} \subseteq \mathrm{Z}$, and $\mathrm{Y}$ and $\mathrm{Z}$ have measure zero.

Or consider the case in which $\mathrm{u}$ is a proper part of $\mathrm{v}$ and $\mathrm{u}$ and $\mathrm{v}$ have the same finite hypervolume. 
or we must resort to infinitesimals. The first horn is uncomfortable because intuitively there should be a measure of the quantity of the most fundamental stuff of which the universe is made. When this stuff was identified with matter the quantity was thought to be mass, but the aether replaces matter, and so hypervolume is the measure in question. It should not, therefore, assign literally zero to any difference. The second horn is also somewhat uncomfortable because we have an intuitive dislike of the (actual) infinity and the (actual) infinitesimal, as shown by the widespread and, I say, deserved, acclaim for the way mathematicians such as Augustin-Loius Cauchy and Karl Weierstrass developed a rigorous theory of the calculus free from infinities and infinitesimals.

This dilemma is not a conclusive refutation, which is why I called its horns uncomfortable not sharp, but, together with the advantage of classical mereology, it provides the case for preferring Arntzenius Continuum over Borel Continuum. In Chapter Three, I shall show that an apparent advantage of Borel Continuum, arising from Chapter Two, is not genuine.

\section{Open Gunk}

A variant on Arntzenius Continuum may be obtained by taking only the non-empty open subsets of $\mathrm{E}$ to represent regions, and once again treating as equivalent two sets that differ only by measure zero. In that case, if $\mathrm{Z}$ is an equivalence class of open sets, $\cup \mathrm{Z} \in \mathrm{Z}$ and $\mathrm{I}$ call the union of an equivalence class of open sets a maximal open set. So the regions may be represented in a one to one fashion by the non-empty maximal open sets. This is a complete Heyting mereology. ${ }^{53}$ I mention it largely to exhibit the range of hypotheses. It is one of a variety of hypotheses in which regions are represented by open sets and in which summation is represented by union. I call these Open Gunk hypotheses. They include Locale Continuum, the hypothesis I have already mentioned as rejected by Hudson because it violates Weak Supplementation. On it the regions

53 The proofs of this result and of the previous result that for any equivalence class $Z, \cup Z \in Z$, rely on the fact that in the topological space of n-tuples of real numbers the union of a set $X$ of open sets is the union of some countable subset of $X$. 
are represented in a one to one way by all the non-empty open subsets of E. I call it Locale Continuum because of its connection with the theory of locales (Johnstone 1982). In Chapter Three I shall explain why I reject them.

The method of representation enables is to consider other hypotheses such as the following.

\section{Sparse Continuum}

On this hypothesis, we start with the set $\mathrm{S}$ of open convex subsets of $\mathrm{E}$ and consider all complements and finite unions of $\mathrm{S}$ as representing regions. More precisely, consider $\mathrm{S}^{*}$ the intersection of all the sets $\mathrm{Z}$ of sets of quadruples that: (1) $S \in Z$; (2) if $X \in Z$ and $Y \in Z$ then $X-Y \in$ $\mathrm{Z}$ and $\mathrm{X} \cap \mathrm{Y} \in \mathrm{Z}$. Then the regions are represented by all and only the non-empty members of $\mathrm{S}^{*}$.

\section{Tarski Continuum}

Finally I should mention Tarski's hypothesis that all and only the nonempty regular open subsets of $E$ represent regions(1956). This is not an Open Gunk hypothesis because summation fails to correspond to union of sets. It is a classical mereological theory.

\section{Curmudgeon variants}

The restriction that all regions are connected may be expressed easily enough if the regions are represented faithfully by open sets. For a nonempty open set is connected if it is not the union of two disjoint nonempty open sets. More generally, a region $u$ is connected unless there is some positive $\delta$ such that $\mathrm{u}$ is the sum of regions $\mathrm{v}$ and $\mathrm{w}$ and no region of diameter less $\delta$ than overlaps both $\mathrm{v}$ and $\mathrm{w}$. So if $\mathrm{X}$ is the set of nonempty sets of quadruples used to represent the regions ignoring curmudgeons, then curmudgeons may represent the regions by $\mathrm{Xc} \subset \mathrm{X}$, where, ifU $\in X, U \in X c$ unless there is some positive $\delta$ and $U$ is the union of $\mathrm{V} \in \mathrm{X}$ and $\mathrm{W} \in \mathrm{X}$ such that no $\mathrm{Y} \in \mathrm{X}$ of diameter less than $\delta$ intersects both $\mathrm{V}$ and $\mathrm{W}$. 
Restrictions on the representation of the whole

I have assumed that $\mathrm{E} \subseteq$. $\mathfrak{R} 4$. A variant is obtained by increasing the number of dimensions from 4 to $\mathrm{k} \geq 9$ and representing curved aether without approximation in $\Re \mathrm{k}$. We can do this by relying on John Nash's Imbedding Theorem (Nash 1956). In that case, $\mathbb{E}$ is a 4-dimensional differentiable manifold. We may use the diameter as defined in $\Re \mathrm{k}$ but the measure is that for the manifold not that for $\mathfrak{R k}$, which would assign zero to œ.

Another restriction is to the union of the boundaries of nonoverlapping polytopic open sets, of the sort used to represent Extended Simples. In that case the aether has only 3 dimensions. We could go further and take $E$ to be the union of the 1 dimensional edges of the polytopes. Yet another restriction, based on discrete Time would be to take $\mathrm{E}$ to be the union of countably many space-like aether hypersurfaces corresponding to a discrete sequence of moments.

\section{Fine structure}

The method of representation directs our attention to the fine structure, if there is any, because that is what the representation ignores. I shall not be concerned much with fine structure because Ockham's Razor inclines me to deny there is any. Moreover, hypotheses that I will be comparing can be stated without commitment to whether or not there is fine structure. It is, however, instructive to state some hypotheses concerning fine structure as part of my overall purpose in this chapter, namely showing that there is a great variety of ways the aether might be, for all we know.

One kind of fine structure would occur if each region $\mathrm{x}$ contains a certain quantity of aether and if $x$ has parts that have the same extension as $x$ but contain only some of $x$ 's aether. In that case even regions that have no proper subregions might fail to be simple having parts consisting of a proportion between 0 and 1 of the aether. This will be briefly considered in Chapter Five.

Another kind of fine structure occurs if the regions are represented, perhaps faithfully, as sets of quadruples of non-standard numbers, say of the form $x+x^{\prime} \imath+x^{\prime} \imath^{2}$ where $x, x^{\prime}$ and $x^{\prime \prime}$ are standard real numbers and $\mathrm{\imath}$ an infinitesimal. Call this the fine representation. Then there is a coarse 
representation obtained by ignoring the infinitesimals. There is an equivalence relation that holds between regions $u$ and $v$ just in case they have the same coarse representation. If there are points, that is regions of zero coarse diameter, then the sum of all the points in a given equivalence class is a maximal point. Consider, for example, the maximal point with coarse representation $\{<0,0,0,0>\}$. Its fine representation is $\left\{<t^{\prime} \mathfrak{\imath}\right.$ $\mathrm{t}^{\prime \prime} \mathrm{l} 2, \mathrm{x}^{\prime} \mathrm{\imath}+\mathrm{x}^{\prime \prime}{ }^{\prime} \mathrm{l} 2, \mathrm{y}^{\prime} \mathrm{\imath}+\mathrm{y}^{\prime \prime} \mathrm{\imath} 2, \mathrm{z}^{\prime} \mathrm{\imath}+\mathrm{z}^{\prime \prime} \mathrm{l} 2>: \mathrm{t}^{\prime}, \mathrm{t}^{\prime \prime}, \mathrm{x}^{\prime}, \mathrm{x}^{\prime \prime}, \mathrm{y}^{\prime}, \mathrm{y}^{\prime \prime}, \mathrm{z}^{\prime}$, and $z^{\prime \prime}$ are standard real numbers $\}$. So in this case the maximal point has the structure of an eight dimensional space. If we allowed powers of 1 up to and including the $\mathrm{kth}$, then the maximal points have $4 \mathrm{k}$ dimensions.

Another way of hypothesising fine structure is to start with a fine representation of the regions as sets of quintuples $<w ; t, x, y, z>$ where $<\mathrm{t}, \mathrm{x}, \mathrm{y}, \mathrm{z}>\in \mathfrak{R} 4$ but $w \in \mathrm{M}$, some set with a topology on it, for instance a manifold. In that case if there are points, then each maximal point has the structure of $\mathrm{M}$, which could be of any number of dimensions. Each maximal point might for instance have the structure of a circle so $w$ is an angle.

This survey of fine structure is not intended as exhaustive but merely sufficient to add to the enormous variety of structures we can conceive of the aether having. It should be noted that if there is fine structure a point is not an atom. Because I shall largely ignore fine structure the important question is not whether the aether is atomic or gunk but whether it has points or not.

\section{Extent.}

I am delaying most considerations of structure in addition to the mereological, but some intuitions about extent and quantity will be required for the next chapter. We may measure the extent of a region by means of a diameter function, which will assign either $\infty$ or a non-negative real number $|\mathrm{x}|$ to each region $\mathrm{x}$. It must satisfy:

Diam 1: If $\mathrm{x} \leq \mathrm{y}$ then $|\mathrm{x}| \leq|\mathrm{y}|$; and

Diam 2: If $x$ and $y$ overlap, and if they have a join $x \vee y$, then $|\mathrm{x} \vee \mathrm{y}| \leq|\mathrm{x}|+|\mathrm{y}|$.

It is worth pausing to think about plausible hypotheses in which the join is not a fusion to check that Diam2 is rightly stated using the join not the 
sum. On the Intervals Only hypothesis about Time, joins are not always fusions but Diam 2 is still intuitively correct, as it would be if, contrary to intuition, we supposed that all regions are convex.

Unless the aether is discrete, there is an argument against treating diameter as a primitive property, analogous to the arguments of Robin le Poidevin (2004) and Tim Maudlin (2007: 86-89) against a primitive relation of distance. The argument is that Diam2 is a necessary constraint on the scalar diameter property, but one that lacks explanation. Necessities should not be multiplied more than is necessary, so we should not treat Diam2 as primitive. My response is that we start with the linear ordering due to a relation of being of greater extent, about which we can say that no part has greater-extent than the whole. ${ }^{54}$ Then, paralleling the methods of measuring extent with rulers, we quantify this obtaining a diameter function (arbitrary up to a scale factor). ${ }^{55}$ Regarding Diam2, suppose we had a measure of extent, call it quasi-diameter, that satisfies Diam1 but not Diam2. Then we could define an associated distance relation between regions $u$ and $v$ as the greatest lower bound of the sums of the quasi-diameters of chains of overlapping regions joining $u$ to $v .^{56}$ Then the diameter of a region $w$ is the least upper bound of the distances between parts of $\mathrm{w}$. This method of characterising the diameter could fail if the distance function assigned zero to regions that did not have zero quasi-diameter, but otherwise we obtain a measure of extent that satisfies Diam1 and Diam2. Because Special Relativity implies that the diameter is frame-relative it is unlikely, though, that diameter, or even the greater extent relation, is a fundamental feature of the aether. So the details of deriving the diameter function as a way of measuring extent

It is a linear ordering in the sense that: (a) it is transitive; and (b) the derived relation of being neither of greater nor of lesser extent is an equivalence relation.

There is a well developed theory of how quantitative scales can be derived from linear orderings given various plausible constraints (Suppes and Zinnes, 1963) (Luce and Suppes 2002).

Each region in the chain overlaps the next, the first overlaps $u$, and the last overlaps v. 
should not concern us too much. For the present it suffices that we have intuitions about diameters.

I define a point as a region of zero diameter, noting that atoms need not be points and points need not be atoms. To ensure that all atoms are points it suffices that the following holds.

Covering: Given any positive integer $\mathrm{N}$ and any region $\mathrm{x}, \mathrm{x}$ is covered by (i.e. is part of the join of) regions of diameter less than $1 / \mathrm{N}$.

Clearly Covering fails for the aether simples posited in the Extended Simples hypothesis.

There are some (mereo-)topological relations that might be considered primitive but may be defined in terms of diameter if there is a diameter function, and if Covering holds. I say that the adjacency or touching relation holds between regions $\mathrm{x}$ and $\mathrm{y}(\mathrm{x} @ \mathrm{y})$ if for every positive integer $\mathrm{N}$ there is some region $\mathrm{z}$ overlapping both $\mathrm{x}$ and $\mathrm{y}$ such that $\mathrm{z}$ has diameter less than $1 / \mathrm{N}$. I say that regions $\mathrm{x}$ and $\mathrm{y}$ are separated if they are not adjacent. I say that $\mathrm{x}$ is an interior part of $\mathrm{y}, \mathrm{x}$ « $\mathrm{y}$ if, for some positive integer $\mathrm{N}$, every region of diameter less than $1 / \mathrm{N}$ that overlaps $\mathrm{x}$ is part of $\mathrm{y}$. It follows that if $\mathrm{x} \ll \mathrm{y}$ and $\mathrm{y}$ and $\mathrm{z}$ are disjoint then $x$ and $z$ are separated. And if $x$ and $z$ are separated then $x$ « $\neg \mathrm{z}$. If the mereology is classical, then every region is a complement, and so $\mathrm{x}$ « $\mathrm{y}$ if and only if $\mathrm{x}$ is separated from $\neg \mathrm{y}$.

Adjacency is an equivalence relation on points. To ensure that the join of an equivalence class of points is a point it suffices to assume that any two regions have a join together with the following:

Remote Parts: Given any region $\mathrm{x}$ and any positive integer $\mathrm{N}$, $\mathrm{x}$ has parts $\mathrm{y}$ and $\mathrm{z}$ such that any region overlapping both $\mathrm{y}$ and $\mathrm{z}$ has diameter at least $|\mathrm{x}|-1 / \mathrm{N}$.

\section{Quantity}

The quantity of a region is measured by its hypervolume, a function assigning either $0, \infty$ or some positive real number to all or some of the regions. It is intuitive that every region has a hypervolume, but this intuition will be delayed until the next chapter. Meanwhile I am permitting 
the Orthodoxy, as I call it, in which the regions correspond in a one to one fashion to all the non-empty sets of coordinate quadruples. Assuming the Axiom of Choice, the Banach Tarski theorem then shows that not every region can be assigned a hypervolume. Therefore, when I state the rules governing hypervolumes I am restricting them, if necessary, to those regions that have a hypervolume (abbreviated to hvol). We have:

Superadditivity: If regions $\mathrm{u}$ and $\mathrm{v}$ are disjoint and $\mathrm{w}$ is a region such that $\mathrm{u}$ and $\mathrm{v}$ are both parts of $\mathrm{w}$, then $\mathrm{hvol}(\mathrm{w}) \geq \mathrm{hvol}(\mathrm{u})+$ hvol(v).

If $\mathrm{u}$ and $\mathrm{v}$ have a sum, $\mathrm{u}+\mathrm{v}$, Subadditivity should also hold, namely that $\operatorname{hvol}(u+v) \leq \operatorname{hvol}(u)+\operatorname{hvol}(v)$.

I note, however, that summation is a rather special operation on regions, and in a survey of hypotheses we should not prematurely assume the existence of sums. In place of Subadditivity I propose therefore:

Approximate Subadditivity: If $\mathrm{u}<\mathrm{v}$, then given any positive integer

$\mathrm{N}$, there is some positive integer $\mathrm{n}$ and pairwise disjoint regions $\mathrm{u} 0, \mathrm{u}_{1}, \ldots, \mathrm{u}_{\mathrm{n}}$ such that $\mathrm{u}_{0}=\mathrm{u}, \mathrm{uj}_{\mathrm{j}}<\mathrm{v}$ and $\sum\left\{\operatorname{hvol}\left(\mathrm{uj}_{\mathrm{j}}\right): \mathrm{j}=0, \ldots\right.$ $, \mathrm{n}\} \geq \operatorname{hvol}(\mathrm{v})-1 / \mathrm{N}$.

\section{Conclusions of survey}

This chapter has shown that there are an enormous variety of available hypotheses about the structure of the aether, between which to choose. My procedure in this work is to begin with some intuitive, considerations and then examine the kinds of extra structure that the aether might be supposed to have, including the structures posited by current physics. My starting point will be to show that we have inconsistent intuitions about the aether and to generate a range of four hypotheses that give up just one intuition each, the exemplars.

\section{Conflicting Intuitions}

In the previous chapter I exhibited the variety of hypotheses about the aether's necessary structure. If there was a well supported unified phys- 